\title{
SVD-AIDED, ITERATIVELY DETECTED SPATIAL DIVISION MULTIPLEXING USING LONG-RANGE CHANNEL PREDICTION
}

\author{
Andreas Ahrens ${ }^{1}$, Wei Liu ${ }^{2}$, Soon Xin $\mathrm{Ng}^{2}$, Volker Kuehn ${ }^{1}$, Lie-Liang Yang ${ }^{2}$ and Lajos Hanzo ${ }^{2}$ \\ ${ }^{1}$ University of Rostock, Institute of Communications, andreas.ahrens@uni-rostock.de \\ ${ }^{2}$ University of Southampton, School of ECS, lh@ecs.soton.ac.uk
}

\begin{abstract}
In this contribution iteratively detected spatial division multiplexing is investigated under the constraint of a fixed data throughput. Existing bit loading and transmit power allocation techniques are often optimized for maintaining both a fixed transmit power and a fixed target bit-error rate, while attempting to maximize the overall datarate, albeit delay-critical real-time interactive applications, such as voice or video transmission, may require a fixed data rate. As an alternative design option, in addition to sophisticated joint bit- and power loading, in this contribution we invoke both coded modulation as well as channel prediction and identify the most beneficial number of modulation signalling levels, while minimizing the bit-error ratio under the constraints of a given fixed throughput. Our performance results show the superiority of bit-interleaved coded modulation using iterative decoding (BICM-ID) against turbo trellis-coded modulation (TTCM), regardless of using idealistic perfect or realistic imperfect channel state information (CSI).
\end{abstract}

Index Terms - Multiple-Input Multiple-Output, Spatial Division Multiplexing, Coded Modulation, Iterative Detection

\section{INTRODUCTION}

Multiple-Input Multiple-Output (MIMO) systems are capable of increasing both the achievable capacity and the integrity of wireless systems $[1,2]$. Unfortunately, their parameters cannot be optimized independently from each other, requiring a careful joint optimization procedure in order to fulfill specific design criteria. Therefore, the most beneficial choice of the number of bits per symbol and the appropriate allocation of the transmit power, combined with powerful joint coding and modulation schemes offers a certain degree of design freedom, which substantially affects the performance of MIMO systems. In general, the appropriate choice of the number of MIMO layers allows us to maximize the system's effective throughput, while maintaining the best possible bit error-ratio (BER). In contrast to using a number of MIMO layers, eigen-beamforming aided schemes have attracted substantial research attenuation, where all available resources are focussed on the strongest eigen-beam [3]. Joint coding and modulation such as Trellis Coded Modulation (TCM) was introduced by Ungerböck in 1982 [4] for non-dispersive Gaussian channels. This concept has motivated intensive research efforts, especially after the conception of turbo codes by Berrou et al. [5], leading to the concept of Turbo TCM (TTCM), which was proposed by Robertson and Wörz [6]. The combination of the turbocoding concept with powerful bandwidth efficient component codes facilitates iterative decoding, and in TTCM symbol-by-symbol MAP components decoders operating in the log domain are used in contrast to the bit-based log-likelihood ratios (LLRs) applied in the bitinterleaved coded modulation using iterative detection (BICM-ID).
In this contribution, the BER performance of eigen-beamforming aided TTCM schemes is studied in comparison to spatial multiplexingstyle MIMOs using the appropriate choice of the number of iteratively detected MIMO layers in terms of the bit error-rate (BER). A two-stage optimization technique is considered. Firstly, the uncoded spatial division multiplexing (SDM) aided MIMO system is optimized, investigating the adaptive allocation of the number of bits to both the modulation and to the SDM scheme at a fixed data rate. Secondly, according to this first stage, the optimized uncoded systems are extended by incorporating both TTCM and BICM-ID as well as pilot-based Minimum Mean Square Error (MMSE) long-range channel prediction, whereby both the uncoded as well as the coded systems are required to support the same user data rate within the same bandwidth.

Against this background, the novel contribution of this paper is that we demonstrate the benefits of amalgamating pilot-based MMSE long-range channel prediction with sophisticated coded modulation as well as with iteratively detected schemes under the constraint of a given data throughput.

This contribution is organized as follow: Section 2 introduces our system model, while the proposed MIMO channel prediction is discussed in Section 3. In Section 4 the channel encoded MIMO system is introduced, while the associated performance results are presented and interpreted in Section 5. Finally, Section 6 provides our concluding remarks.

\section{SYSTEM OVERVIEW}

Consider a system employing $M_{\mathrm{T}}$ transmit and $M_{\mathrm{R}}$ receive antennas for communicating over flat Rayleigh fading channels. The transmission system can be modelled by

$$
\mathbf{u}=\mathbf{H} \cdot \mathbf{c}+\mathbf{w} .
$$

In (1), $\mathbf{u}$ is the $\left(M_{\mathrm{R}} \times 1\right)$ received vector at the symbol instant $k$, $\mathbf{c}$ is the $\left(M_{\mathrm{T}} \times 1\right)$ transmitted signal vector containing the complex input symbols and $\mathbf{w}$ is the $\left(M_{\mathrm{R}} \times 1\right)$ vector of the Additive, White Gaussian Noise (AWGN) having a variance of $U_{\mathrm{R}}^{2}$ for both the real and imaginary parts. The fading channel coefficient between the $m_{\mathrm{T}}$-th transmit antenna and the $m_{\mathrm{R}}$-th receive antenna is given by

$$
h_{m_{\mathrm{R}} m_{\mathrm{T}}}=\frac{1}{\sqrt{N_{\mathrm{L}}}} \sum_{\mu=1}^{N_{\mathrm{L}}} a_{\mu} \mathrm{e}^{-\mathrm{j} 2 \pi f_{\mathrm{d}} T_{\mathrm{S}} \cos \left(\phi_{\mu}\right)},
$$

where $a_{\mu}$ is a complex-valued Gaussian random variable with zero mean and unit variance, $\phi_{\mu}$ is uniformly distributed over $(0,2 \pi], f_{\mathrm{d}}$ is the maximum Doppler frequency, $T_{\mathrm{s}}$ is the symbol period and $N_{\mathrm{L}}$ is the number of scatterers. 
The interference between the different antennas data streams, which is introduced by the non-diagonal channel matrix $\mathbf{H}$, requires powerful interference cancellation strategies. A popular technique is based on the Singular Value Decomposition (SVD) of the system matrix $\mathbf{H}$, which can be written as ${ }^{1}$

$$
\mathbf{H}=\mathbf{S} \cdot \mathbf{V} \cdot \mathbf{D}^{\mathrm{H}},
$$

where $\mathbf{S}$ and $\mathbf{D}^{\mathrm{H}}$ are unitary matrices and $\mathbf{V}$ is a real-valued diagonal matrix of the positive square roots of the eigenvalues of the matrix $\mathbf{H}^{\mathrm{H}} \mathbf{H}$ sorted in descending order. The SDM MIMO input data vector $\mathbf{c}$ is now multiplied by the matrix $\mathbf{D}$ before transmission. In turn, the receiver multiplies the received vector $\mathbf{u}$ by the matrix $\mathbf{S}^{\mathrm{H}}$. Thereby neither the transmit power nor the noise power is enhanced. When taking into account Eq. (3), the overall transmission relationship is formulated as

$$
\mathbf{y}=\mathbf{S}^{\mathrm{H}} \cdot \mathbf{u}=\mathbf{S}^{\mathrm{H}}(\mathbf{H} \cdot \mathbf{D} \cdot \mathbf{c}+\mathbf{w})=\mathbf{V} \cdot \mathbf{c}+\tilde{\mathbf{w}},
$$

which explicitly indicates that the channel matrix $\mathbf{H}$ was transformed into independent, non-interfering layers represented by the diagonal matrix $\mathbf{V}$, again, constituted by the ordered positive square roots of the eigenvalues of $\mathbf{H}^{\mathrm{H}} \mathbf{H}$.

In general, the quality of data transmission can be informally assessed by using the half vertical eye opening and the noise power at the detector's input [7]. The resultant signal-to-noise ratio (SNR) per quadrature component becomes [8]

$$
\varrho=\frac{(\text { Half vertical eye opening })^{2}}{\text { Noise Power }}=\frac{\left(U_{\mathrm{A}}\right)^{2}}{\left(U_{\mathrm{R}}\right)^{2}},
$$

which is often used as a quality parameter $[8,7]$. The relationship between the SNR $\varrho=U_{\mathrm{A}}^{2} / U_{\mathrm{R}}^{2}$ and the bit-error probability evaluated for AWGN channels and $M$-ary Quadrature Amplitude Modulation (QAM) is given by $[9,10]$

$$
P_{\mathrm{b}}=\frac{2}{\log _{2}(M)}\left(1-\frac{1}{\sqrt{M}}\right) \operatorname{erfc}\left(\sqrt{\frac{\varrho}{2}}\right) .
$$

When applying the proposed system structure, the SVD-based interlayer MIMO equalization leads to different eye opening per MIMO layer and per transmitted symbol block according to

$$
U_{\mathrm{A}}^{(\mu)}=\sqrt{\xi_{\mu}} \cdot U_{\mathrm{s} \mu},
$$

where $U_{\mathrm{s} \mu}$ denotes the half-level transmit amplitude assuming $M_{\mu}$ ary QAM and $\sqrt{\xi_{\mu}}$ represents the positive square roots of the eigenvalues of the matrix $\mathbf{H}^{\mathrm{H}} \mathbf{H}$.

Considering QAM constellations, the average transmit power $P_{\mathrm{s} \mu}$ per MIMO layer may be expressed as [11, 12]:

$$
P_{\mathrm{s} \mu}=\frac{2}{3} U_{\mathrm{s} \mu}^{2}\left(M_{\mu}-1\right) .
$$

Using the parallel transmission of $L \leq \min \left(M_{\mathrm{T}}, M_{\mathrm{R}}\right)$ MIMO layers, the overall mean transmit power becomes

$$
P_{\mathrm{s}}=\sum_{\mu=1}^{L} P_{\mathrm{s} \mu}
$$

\footnotetext{
${ }^{1}$ The transpose and conjugate transpose (Hermitian) of $\mathbf{D}$ are denoted by $\mathbf{D}^{\mathrm{T}}$ and $\mathbf{D}^{\mathrm{H}}$, respectively.
}

Table 1. QAM constellations

\begin{tabular}{ccccc}
\hline throughput & layer 1 & layer 2 & layer 4 & layer 4 \\
\hline 4 bit/s/Hz & 16 & 0 & 0 & 0 \\
$\mathbf{4}$ bit/s/Hz & $\mathbf{4}$ & $\mathbf{4}$ & $\mathbf{0}$ & $\mathbf{0}$ \\
\hline $6 \mathrm{bit} / \mathrm{s} / \mathrm{Hz}$ & 64 & 0 & 0 & 0 \\
$\mathbf{6} \mathbf{~ b i t} / \mathbf{s} / \mathbf{H z}$ & $\mathbf{1 6}$ & $\mathbf{4}$ & $\mathbf{0}$ & $\mathbf{0}$ \\
$6 \mathrm{bit} / \mathrm{s} / \mathrm{Hz}$ & 4 & 4 & 4 & 0 \\
\hline $8 \mathrm{bit} / \mathrm{s} / \mathrm{Hz}$ & 256 & 0 & 0 & 0 \\
$8 \mathrm{bit} / \mathrm{s} / \mathrm{Hz}$ & 64 & 4 & 0 & 0 \\
$\mathbf{8} \mathbf{~ b i t} / \mathbf{s} / \mathbf{H z}$ & $\mathbf{1 6}$ & $\mathbf{4}$ & $\mathbf{4}$ & $\mathbf{0}$ \\
$8 \mathrm{bit} / \mathrm{s} / \mathrm{Hz}$ & 4 & 4 & 4 & 4 \\
\hline $12 \mathrm{bit} / \mathrm{s} / \mathrm{Hz}$ & 4096 & 0 & 0 & 0 \\
$12 \mathrm{bit} / \mathrm{s} / \mathrm{Hz}$ & 512 & 16 & 0 & 0 \\
$12 \mathrm{bit} / \mathrm{s} / \mathrm{Hz}$ & 64 & 64 & 0 & 0 \\
$\mathbf{1 2} \mathbf{b i t} / \mathbf{s} / \mathbf{H z}$ & $\mathbf{6 4}$ & $\mathbf{1 6}$ & $\mathbf{4}$ & $\mathbf{0}$ \\
$12 \mathrm{bit} / \mathrm{s} / \mathrm{Hz}$ & 64 & 4 & 4 & 4 \\
$12 \mathrm{bit} / \mathrm{s} / \mathrm{Hz}$ & 16 & 16 & 16 & 0 \\
$12 \mathrm{bit} / \mathrm{s} / \mathrm{Hz}$ & 16 & 16 & 4 & 4 \\
\hline
\end{tabular}

where $\min \left(M_{\mathrm{T}}, M_{\mathrm{R}}\right)$ defines the number of readily separable lay$\mathrm{ers}^{2}$. In order to transmit at a fixed data rate while maintaining the best possible integrity, i. e. BER, an appropriate number of MIMO layers has to be used, which depends on the specific QAM constellation size, as detailed in Tab. 1. The bit-error probability per QAM symbol after SVD is given by [7]

$$
P_{\mathrm{b} \mu}=\frac{2\left(1-\frac{1}{\sqrt{M_{\mu}}}\right)}{\log _{2}\left(M_{\mu}\right)} \operatorname{erfc}\left(\sqrt{\frac{\xi_{\mu}}{2}} \cdot \frac{U_{\mathrm{s} \mu}}{U_{\mathrm{R}}}\right) .
$$

The resultant average bit-error probability assuming different QAM constellation sizes per MIMO layer is given by:

$$
P_{\mathrm{b}, \text { block }}=\frac{1}{\sum_{\nu=0}^{L} \log _{2}\left(M_{\nu}\right)} \sum_{\mu=0}^{L} \log _{2}\left(M_{\mu}\right) P_{\mathrm{b} \mu} .
$$

When considering time-variant channel conditions, rather than an AWGN channel, the BER can be derived by considering the approximately constant transmission blocks SNRs.

\section{MIMO CHANNEL PREDICTION}

The employment of a reliable channel predictor is imperative for achieving low-SNR, high-throughput operations. In this contribution, we will employ a low-complexity Minimum Mean Square Error (MMSE) based pilot-symbol aided MIMO long-range channel predictor $[14,15,16]$. Both transmit and receive buffers are used for buffering an encoded frame, which is partitioned into shorter subframes and $M_{\mathrm{T}}$ number of pilot symbols are attached to each subframe at its beginning, as seen in Fig. 1. A shorter subframe length is expected to increase the accuracy of the channel predictor, but

\footnotetext{
${ }^{2}$ It is worth noting that with the aid of powerful non-linear near Maximum Likelihood (ML) sphere decoders it is possible to separate $M_{\mathrm{R}}>M_{\mathrm{T}}$ number of layers [13].
} 
naturally, it also imposes a higher pilot symbol overhead. During the transmission of the pilot symbols only one transmit antenna is activated for the corresponding symbol period, while the remaining transmit antennas are deactivated as seen in Fig. 1. This allows us to avoid having a low pre-antenna power and hence improves the achievable channel estimation accuracy.

Let us denote the index of the subframe by the subscript $l$ and the time index by $m_{\mathrm{T}}$ for reasons of brevity in this section. For the $l$ th subframe transmitted at time instant $m_{\mathrm{T}}\left(1 \leq m_{\mathrm{T}} \leq M_{\mathrm{T}}\right)$, the signal $y_{m_{\mathrm{R}}}\left[l, m_{\mathrm{T}}\right]$ received by the $m_{\mathrm{R}}$-th $\left(1 \leq m_{\mathrm{R}} \leq M_{R}\right)$ receiver antenna is given by

$$
y_{m_{\mathrm{R}}}\left[l, m_{\mathrm{T}}\right]=h_{m_{\mathrm{R}} m_{\mathrm{T}}}\left[l, m_{\mathrm{T}}\right] \cdot c_{\mathrm{p}}+\tilde{w}_{m_{\mathrm{R}}}\left[l, m_{\mathrm{T}}\right] \text {, }
$$

where $h_{m_{\mathrm{R}} m_{\mathrm{T}}}\left[l, m_{\mathrm{T}}\right]$ represents the fading channel coefficient between the $m_{\mathrm{T}}$-th transmit antenna and the $m_{\mathrm{R}}$-th receive antenna for the $l$ th subframe at time instant $m_{\mathrm{T}}$, while $c_{\mathrm{p}}$ represents the pilot symbol, which is assumed to be the same for all transmit antennas and for all subframes. Furthermore, $\tilde{w}_{m_{\mathrm{R}}}\left[l, m_{\mathrm{T}}\right]$ is the AWGN contribution at the $m_{\mathrm{R}}$ th receiver antenna for the $l$ th subframe at time instant $m_{\mathrm{T}}$. With the aid of the $M_{\mathrm{T}}$-by- $M_{\mathrm{T}}$ pilot symbol matrix seen in Fig. 1, the $\left(M_{\mathrm{R}} \times M_{\mathrm{T}}\right)$-dimensional MIMO channel prediction problem is decomposed into a single-input single-output (SISO) channel prediction scenario, where any classic SISO channel prediction algorithm can be applied [17]. In this contribution, MMSE based narrowband channel prediction is invoked [17]. Specifically, we construct the following $p$-dimensional vector:

$$
\mathbf{y}_{m_{\mathrm{R}}}\left[l, m_{\mathrm{T}}\right]=\left(y_{m_{\mathrm{R}}}\left[l-p+1, m_{\mathrm{T}}\right], \cdots, y_{m_{\mathrm{R}}}\left[l, m_{\mathrm{T}}\right]\right)^{\mathrm{T}} .
$$

If we assign the value of " +1 " to each pilot symbol $c_{\mathrm{p}}$, then the channel coefficient corresponding to the $m_{\mathrm{T}}$-th pilot symbol of the $(l+1)$-th subframe is given by the corresponding received pilot symbol $y_{m_{\mathrm{R}}}\left[l+1, m_{\mathrm{T}}\right]=h_{m_{\mathrm{R}}}\left[l+1, m_{\mathrm{T}}\right] \cdot(+1)$ in the absence of noise. Hence, the predicted channel coefficient corresponding to the $m_{\mathrm{T}}$-th pilot symbol for the $(l+1)$-th subframe, namely $\hat{h}_{m_{\mathrm{R}} m_{\mathrm{T}}}\left[l+1, m_{\mathrm{T}}\right]$, can be estimated based on the received pilot symbol vector of the $l$-th subframe as [15]:

$$
\hat{h}_{m_{\mathrm{R}} m_{\mathrm{T}}}\left[l+1, m_{\mathrm{T}}\right]=\mathbf{d}_{0} \mathbf{y}_{m_{\mathrm{R}}}\left[l, m_{\mathrm{T}}\right],
$$

where $\mathbf{d}_{0}$ is formulated as:

$$
\mathbf{d}_{0}=\mathbf{R}_{\mathbf{y}_{m_{\mathrm{R}}}\left[l, m_{\mathrm{T}}\right]}^{-1} \mathbf{r}_{\mathbf{y}_{m_{\mathrm{R}}}\left[l, m_{\mathrm{T}}\right] y_{m_{\mathrm{R}}}\left[l+1, m_{\mathrm{T}}\right]},
$$

where $\mathbf{R}_{\mathbf{y}_{m_{\mathrm{R}}}\left[l, m_{\mathrm{T}}\right]}$ is the $(p \times p)$-dimensional autocorrelation matrix of $\mathbf{y}_{m_{\mathrm{R}}}\left[l, m_{\mathrm{T}}\right]$, which is given by [15]:

$$
\mathbf{R}_{\mathbf{y}_{m_{\mathrm{R}}}\left[l, m_{\mathrm{T}}\right]}=\mathbf{E}\left[\mathbf{y}_{m_{\mathrm{R}}}\left[l, m_{\mathrm{T}}\right] \mathbf{y}_{m_{\mathrm{R}}}^{\mathrm{H}}\left[l, m_{\mathrm{T}}\right]\right] .
$$

Furthermore, $\mathbf{r}_{\mathbf{y}_{m_{\mathrm{R}}}\left[l, m_{\mathrm{T}}\right] y_{m_{\mathrm{R}}}\left[l+1, m_{\mathrm{T}}\right]}$ is the $p$-dimensional crosscorrelation vector recorded for $\mathbf{y}_{m_{\mathrm{R}}}\left[l, m_{\mathrm{T}}\right]$ and $y_{m_{\mathrm{R}}}\left[l+1, m_{\mathrm{T}}\right]$, which is given by [15]:

$$
\mathbf{r}_{\mathbf{y}_{m_{\mathrm{R}}}\left[l, m_{\mathrm{T}}\right] y_{m_{\mathrm{R}}}\left[l+1, m_{\mathrm{T}}\right]}=\mathbf{E}\left[\mathbf{y}_{m_{\mathrm{R}}}^{*}\left[l, m_{\mathrm{T}}\right] y_{m_{\mathrm{R}}}\left[l+1, m_{\mathrm{T}}\right]\right] .
$$

Finally, the predicted channel coefficients corresponding to the data symbol can be obtained with the aid of interpolation. In this case, the predicted channel coefficients corresponding to the data symbols between two pilots as seen in Fig. 1 can be obtained with the aid of interpolation [16]. For example, if a pilot symbol is inserted every 50 data symbols, with the aid of interpolating between the pilots we can readily derive the complex channel coefficients of the 49 data symbols. Low- complexity linear interpolation was employed.
Using the predicted channel coefficients, the SVD of the predicted system matrix $\widehat{\mathbf{H}}$ results in

$$
\widehat{\mathbf{H}}=\widehat{\mathbf{S}} \cdot \widehat{\mathbf{V}} \cdot \widehat{\mathbf{D}}^{\mathrm{H}} .
$$

Assuming that the estimated matrix $\widehat{\mathbf{D}}$ is known at the transmitter side as well as $\widehat{\mathbf{S}}$ at the receiver side, the overall transmission chain may be characterized as:

$$
\widehat{\mathbf{y}}=\widehat{\mathbf{S}}^{\mathrm{H}} \cdot \mathbf{u}=\widehat{\mathbf{S}}^{\mathrm{H}}(\mathbf{H} \cdot \widehat{\mathbf{D}} \cdot \mathbf{c}+\mathbf{w}) .
$$

In comparisons to perfect channel estimations, the channel matrix $\mathbf{H}$ can no longer be decomposed into a system with non-interfering layers, since the resultant matrix $\widehat{\mathbf{S}}^{\mathrm{H}} \mathbf{H} \cdot \widehat{\mathbf{D}}$ is no longer a diagonal matrix.

\section{CODED MIMO SYSTEM}

The coded modulation transmitter's structure is depicted in Fig. 2. The encoder employs a rate $R=1 / 2$ nonrecursive, Non-Systematic (NSC) convolutional code using generator polynomials of $(7,5)$ in octal represention. The uncoded information is organized in transmission blocks of $N_{\mathrm{a}}$ bits, consisting of at least 1000 bits, depending on the specific QAM constellation used. The data blocks a are encoded and result in the block $\mathbf{b}$ consisting of $N_{\mathrm{b}}=2 N_{\mathrm{a}}+4$ encoded bits, including 4 termination bits. The encoded bits are interleaved using a random interleaver and stored in the interleaved vector $\tilde{\mathbf{b}}$. The encoded and interleaved bits are then mapped to the MIMO layers. The task of the multiplexer and buffer block of Fig. 2 is to divide the vector of encoded and interleaved information bits $\tilde{\mathbf{b}}$ into subvectors $\left(\tilde{\mathbf{b}}_{\mathbf{1}}, \tilde{\mathbf{b}}_{\mathbf{2}}, \cdots, \tilde{\mathbf{b}}_{\mathbf{n}}\right)$, each consisting of 4,8 or 12 bits according to the specific QAM constellation of Tab. 1. The individual binary data vectors $\tilde{\mathbf{b}}_{\mu}$, for $\mu=1,2, \cdots n$, are then mapped to the QAM symbols $c_{\mu}$ according to the specific mapper used. The iterative de-

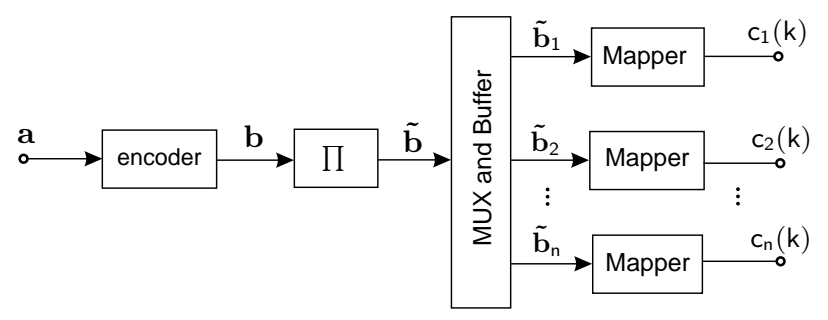

Fig. 2. The channel-encoded MIMO transmitter's structure

modulator's structure is shown in Fig. 3, while a detailed schematic of the soft demapper structure is portrayed in Fig. 4. When using the iteration index $\nu$, the first iteration of $\nu=1$ commences with the soft-demapper delivering the $N_{\mathrm{b}}$ Log-Likelihood Ratios (LLRs) $L_{2}^{(\nu=1)}(\tilde{\mathbf{b}})$ of the encoded and interleaved information bits, whose de-interleaved version $L_{\mathrm{a}, 1}^{(\nu=1)}(\mathbf{b})$ represents the input of the convolutional decoder, as depicted in Fig. 3. This channel decoder provides the estimates $L_{1}^{(\nu=1)}(\mathbf{a})$ of the original uncoded information bits as well as the LLRs of the $N_{\mathrm{b}}$ NSC-encoded bits in the form of

$$
L_{1}^{(\nu=1)}(\mathbf{b})=L_{\mathrm{a}, 1}^{(\nu=1)}(\mathbf{b})+L_{\mathrm{e}, 1}^{(\nu=1)}(\mathbf{b}) .
$$

As seen in Fig. 3 and Eq. (20), the LLRs of the NSC-encoded bits consist of the receiver's input signal itself plus the extrinsic information $L_{\mathrm{e}, 1}^{(\nu=1)}(\mathbf{b})$, which is generated by subtracting $L_{\mathrm{a}, 1}^{(\nu=1)}(\mathbf{b})$ 


\begin{tabular}{|c|c|c|c|c|c|c|c|c|c|c|c|c|c|c|c|c|c|c|c|c|c|}
\hline \multirow{4}{*}{$\begin{array}{l}\text { Number of } \\
\text { transmit } \\
\text { antennas }\end{array}$} & $\mathrm{P}$ & 0 & . & 0 & $\mathrm{D}$ & . & $\mathrm{D}$ & $\mathrm{P}$ & 0 & . & 0 & $\mathrm{D}$ & . & $\mathrm{D}$ & $\mathrm{P}$ & 0 & . & 0 & $\mathrm{D}$ & & $\mathrm{D}$ \\
\hline & 0 & $\mathrm{P}$ & 尓 & $\vdots$ & $\mathrm{D}$ & . & $\mathrm{D}$ & 0 & $\mathrm{P}$ & $\ddots$ & $\vdots$ & $\mathrm{D}$ & $\cdots$ & $\mathrm{D}$ & 0 & $\mathrm{P}$ & $\ddots$ & $\vdots$ & $\mathrm{D}$ & & $\mathrm{D}$ \\
\hline & : & $\ddots$ & $\ddots$ & 0 & $\mathrm{D}$ & . & $\mathrm{D}$ & $\vdots$ & $\ddots$ & $\ddots$ & 0 & $\mathrm{D}$ & . & $\mathrm{D}$ & $\vdots$ & $\ddots$ & $\cdot$ & 0 & $\mathrm{D}$ & & $\mathrm{D}$ \\
\hline & 0 & . & 0 & $\mathrm{P}$ & $\mathrm{D}$ & . & $\mathrm{D}$ & 0 & . . & 0 & $\mathrm{P}$ & $\mathrm{D}$ & . & $\mathrm{D}$ & 0 & . & 0 & $\mathrm{P}$ & D & & $\mathrm{D}$ \\
\hline
\end{tabular}

Subframe length
Frame length (a frame of two subframes)

Fig. 1. The MIMO transmission format, where the notations $P, 0$ and $D$ denote the pilot symbol, zero-energy symbol and data symbol, respectively. The first data symbol column in each subframe is the beamforming transmission vector corresponding to the reference symbol of the differential encoder.

from $L_{1}^{(\nu=1)}(\mathbf{b})$. The appropriately ordered, i. e. interleaved extrinsic LLRs are fed back as a priori information $L_{\mathrm{a}, 2}^{(\nu=2)}(\tilde{\mathbf{b}})$ to the soft demapper of Fig. 3 for the second iteration, and so on. Following the detailed schematic of the soft-demapper in Fig. 4, the $N_{\mathrm{b}}$ LLRs $L_{2}^{(\nu)}(\tilde{\mathbf{b}})$ are composed of the subvectors and result in $\left(L_{2}^{(\nu)}\left(\tilde{\mathbf{b}}_{1}\right), L_{2}^{(\nu)}\left(\tilde{\mathbf{b}}_{2}\right), \cdots, L_{2}^{(\nu)}\left(\tilde{\mathbf{b}}_{n}\right)\right)$, each consisting of 4,8 or 12 elements according to the specific QAM constellation of Tab. 1. Each vector $L_{2}^{(\nu)}\left(\tilde{\mathbf{b}}_{\mu}\right)$ associated with $\mu=1,2, \cdots, n$ is generated by the soft demapper from the MIMO channel's output $y_{\mu}$ assuming perfect channel estimation according to (4), or alternatively from $\widehat{y}_{\mu}$ assuming imperfect channel estimation according to (19), which is then combined with the a-priori information $L_{\mathrm{a}, 2}^{(\nu)}\left(\tilde{\mathbf{b}}_{\mu}\right)$ provided by the channel decoder. After the first iteration, this a-priori information accrues from the $N_{\mathrm{b}}$ LLRs $L_{\mathrm{a}, 2}^{(\nu)}(\tilde{\mathbf{b}})$, which are again decomposed into the subvectors $\left(L_{\mathrm{a}, 2}^{(\nu)}\left(\tilde{\mathbf{b}}_{1}\right), L_{\mathrm{a}, 2}^{(\nu)}\left(\tilde{\mathbf{b}}_{2}\right), \cdots, L_{\mathrm{a}, 2}^{(\nu)}\left(\tilde{\mathbf{b}}_{n}\right)\right)$ each consisting of 4,8 or 12 elements.

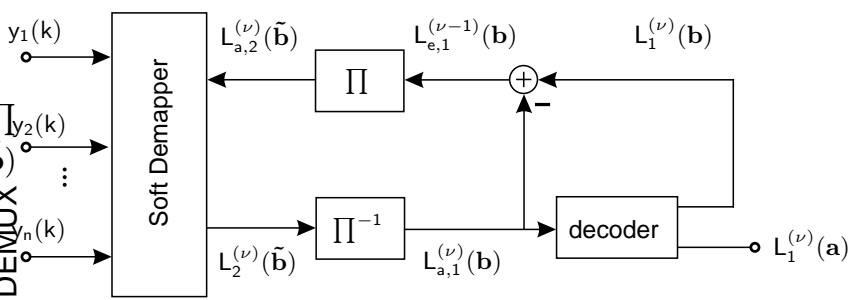

Fig. 3. Iterative demodulator structure

The achievable performance of the iterative decoder is substantially affected by the specific mapping of the bits to both the QAM symbols as well as to the MIMO layers. While the employment of the classic Gray-mapping is appropriate in the absence of a priori information, the availability of a priori information in iteratively detected schemes requires an exhaustive search for finding the best non-Gray - synonymously also referred to as anti-Gray - mapping scheme [18]. Two examples of the investigated 16-QAM mapping schemes are shown in Fig. 5, which have been used in the BICM-ID schemes in [18]

\section{RESULTS}

\subsection{Uncoded transmission assuming perfect CSI}

The resultant BER curves are depicted in Fig. 6, 7, 8 and 9 for the different QAM constellation sizes and MIMO configurations of Tab. 1, when transmitting at a bandwidth efficiency of $12 \mathrm{bit} / \mathrm{s} / \mathrm{Hz}, 8 \mathrm{bit} / \mathrm{s} / \mathrm{Hz}$

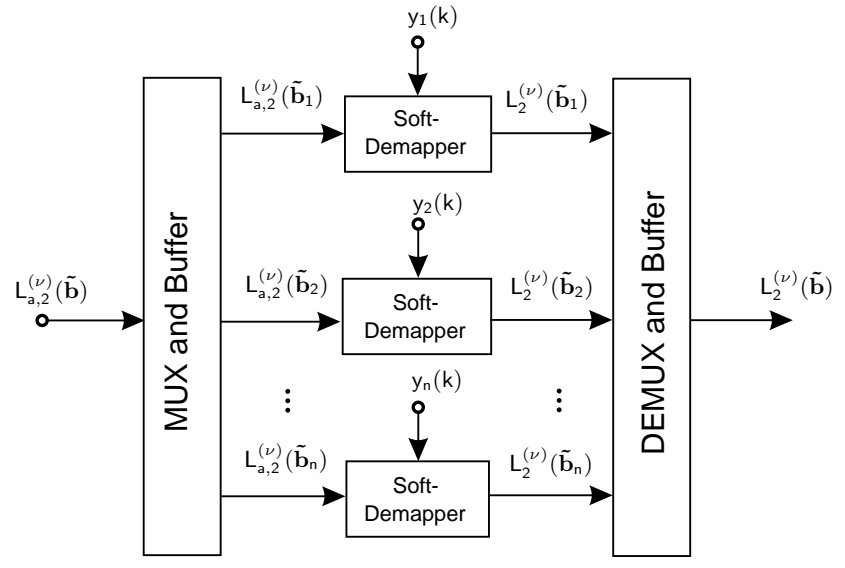

Fig. 4. Detailed soft demapper demodulator structure

and $6 \mathrm{bit} / \mathrm{s} / \mathrm{Hz}$, respectively. Our goal is to find that specific combination of the QAM mode and the number of MIMO layers, which gives the best possible BER performance at a given fixed bit/s/Hz bandwidth efficiency. In contrast to the eigen-beamforming scheme, the total transmit power was uniformly distributed over the MIMO layers used.

\subsection{Iteratively detected MIMO systems}

The attainable BER performance is characterized in Fig. 10 for the half-rate, constraint-length $K=3$ NSC code assuming an effective user throughput of $4 \mathrm{bit} / \mathrm{s} / \mathrm{Hz}$ and compared to that of the TTCM

\begin{tabular}{|c|c|c|c|c|c|c|c|}
\hline \multicolumn{4}{|c|}{$\triangle Q$} & \multicolumn{4}{|c|}{$\uparrow Q$} \\
\hline 1011 & 1001 & $0 \dot{001}$ & 0011 & 1000 & $\dot{1011}$ & 1100 & 1111 \\
\hline $10 \dot{10}$ & 1000 & $0 \dot{000}$ & $0 \dot{0} 10$ & 0001 & $00 \dot{10}$ & $0 \dot{101}$ & $0 i_{10}$ \\
\hline 1110 & 1100 & $0 i 00$ & $0 i 10$ & 0ioo & 0111 & 0000 & 00011 \\
\hline 1111 & 1101 & & $0 i 11$ & 1101 & 1110 & $\dot{1001}$ & 1010 \\
\hline
\end{tabular}

Fig. 5. 16-QAM mapping schemes (left: Gray-coding, right: antiGray coding) 


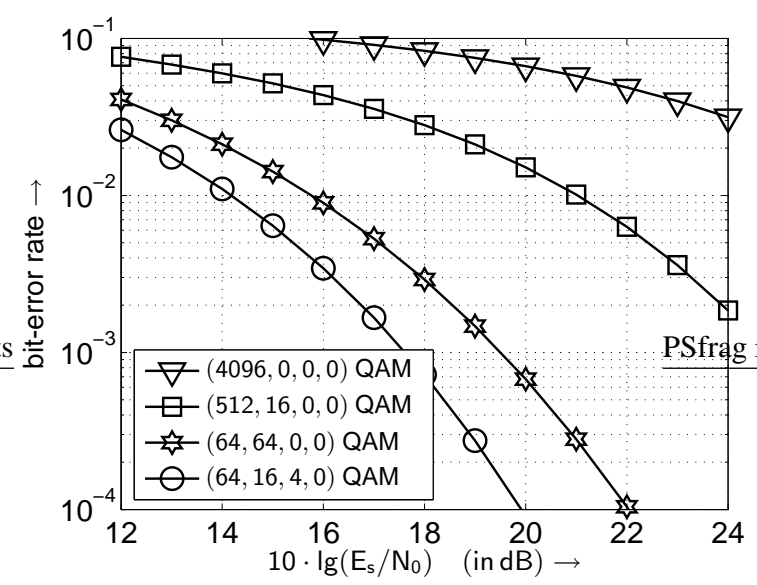

Fig. 6. BER when using the MIMO configurations introduced in Tab. 1 and transmitting $12 \mathrm{bit} / \mathrm{s} / \mathrm{Hz}$ over uncorrelated non-frequency selective channels

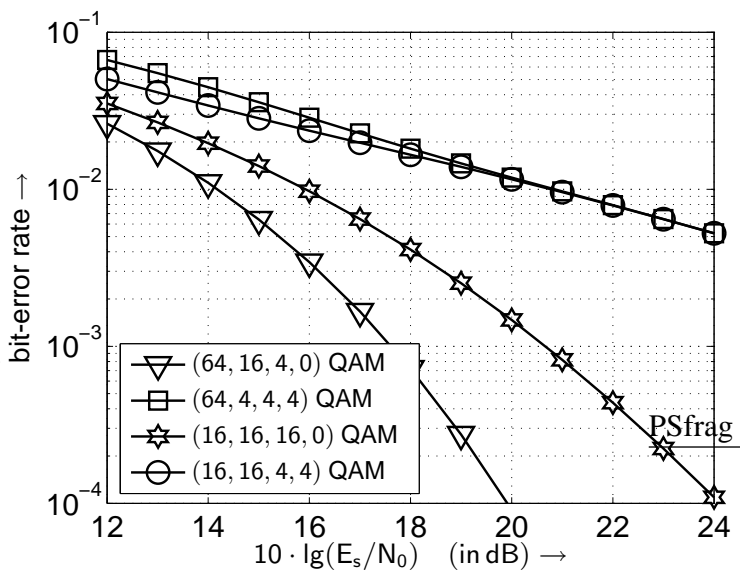

Fig. 7. BER when using the MIMO configurations introduced in Tab. 1 and transmitting $12 \mathrm{bit} / \mathrm{s} / \mathrm{Hz}$ over uncorrelated non-frequency selective channels

scheme at a given fixed user throughput of $4 \mathrm{bit} / \mathrm{s} / \mathrm{Hz}$, when applying a subframe length of 100 . Using the best QAM constellations, the coded scenario is characterized employing the $(16,4,4,0)$ QAM constellations of Tab. 1, as seen in Fig. 8.

\subsection{TTCM}

A TTCM scheme employing two memory-3 (8-states) constituent codes and 4 iterations was used. The parity check polynomial in octal notation is given by $(11,2,4,10,0)$. When using imperfect CSI, the subframe length was again set to 100 . The mapper used is Set-Partitioning based on 32-QAM. Since TTCM is a symbol-based scheme, we cannot readily use iterative detection between the TTCM decoder and the single-layer beamforming based soft demapper. As we can see from Fig. 10, when employing imperfectly predicted CSI, the symbol-based TTCM scheme suffers from a higher BER degradation compared to the bit-based coded scheme.

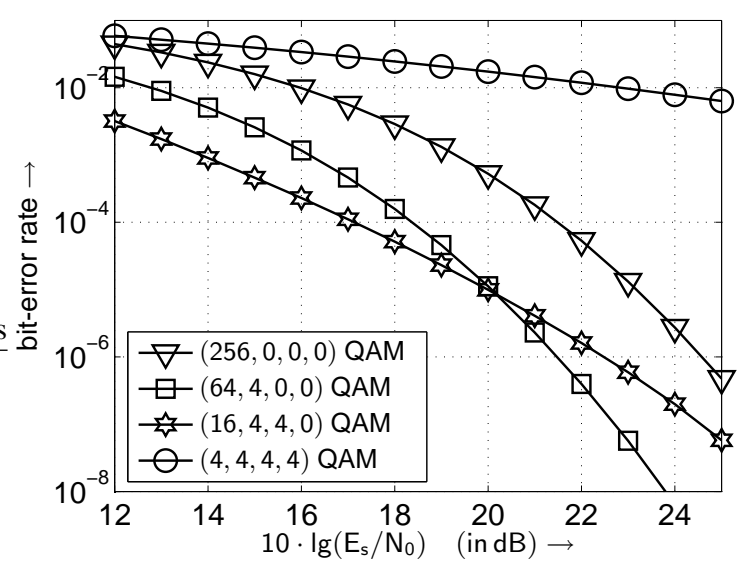

Fig. 8. BER when using the MIMO configurations introduced in Tab. 1 and transmitting 8 bit/s/Hz over uncorrelated non-frequency selective channels

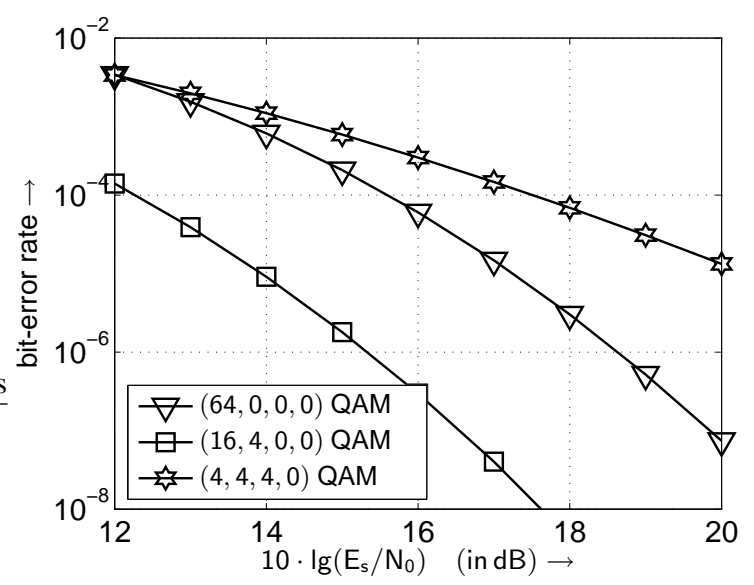

Fig. 9. BER when using the MIMO configurations introduced in Tab. 1 and transmitting 6 bit/s/Hz over uncorrelated non-frequency selective channels

\section{CONCLUSION}

The choice of the number of bits per symbol, the number of MIMO layers, the bit-to-symbol and the bit-to-MIMO-layer mapping scheme combined with the appropriate allocation of the transmit power and error correcting codes substantially affects the performance. The $E_{\mathrm{s}} / N_{0}$ value required by each scheme at BER $10^{-4}$ was extracted from Fig. 6-9 and the best systems are shown in bold font in Tab. 1.

The combination of turbo-codes with powerful bandwidth efficient component codes allows the construction of efficient transmission schemes, whereby the bit-based exchange of the LLRs applied in the BICM-ID system shows a superiority over the symbol-based LLRs applied in the TTCM. 


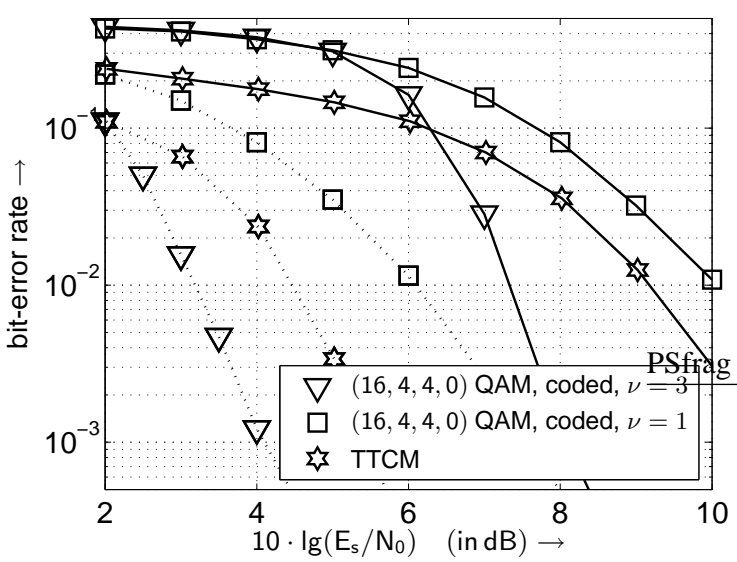

Fig. 10. BER with perfect CSI (dotted line) and imperfect CSI (solid line) assuming an effective user throughput of $4 \mathrm{bit} / \mathrm{s} / \mathrm{Hz}$, Anti-Gray mapping and a normalized Doppler frequency of $f_{\mathrm{d}} T_{\mathrm{s}}=10^{-3}$

\section{Acknowledgement}

The financial support of the EU and of the Royal Society, as well as of the EPSRC, UK is gratefully acknowledged.

\section{REFERENCES}

[1] A. Hottinen, O. Tirkkonen, and R. Wichmann, Multi-antenna Transceiver Techniques for $3 G$ and Beyond., Wiley, West Sussex, 2003.

[2] L. Zheng and D. N. T. Tse, "Diversity and Multiplexing: A Fundamental Tradeoff in Multiple-Antenna Channels.," IEEE Transactions on Information Theory, vol. 49, no. 5, pp. 1073 1096, May 2003.

[3] D. P. Palomar, J. M. Cioffi, and M. A. Lagunas, "Joint TxRx Beamforming Design for Multicarrier MIMO Channels: A Unified Framework for Convex Optimization.," IEEE Transactions on Signal Processing, vol. 51, no. 9, pp. 2381-2401, September 2003.

[4] G. Ungerböck, "Channel coding with multilevel/phase signals," IEEE Transactions on Information Theory, vol. 28, pp. 55-67, January 1982.

[5] C. Berrou, A. Glavieux, and P. Thitimajshima, "Near shannon limit error-correcting coding and decoding: Turbo codes.," in IEEE International Conference on Communications, 1993, pp. 1064-1070.

[6] P. Robertson and T. Wörz, "Bandwidth-efficient turbo trelliscoded modulation using punctured component codes," IEEE Journal on Selected Areas in Communications, vol. 16, no. 2, pp. 206-218, Februar 1998

[7] A. Ahrens and C. Lange, "Transmit Power Allocation in SVD Equalized Multicarrier Systems," International Journal of Electronics and Communications (AEÜ), vol. 61, no. 1, pp. 51-61, 2007.

[8] A. Ahrens and C. Lange, "Equalizer Design for Multicarrier Transmission Systems.," in Nordic Shortwave Conference (Nordic HF), Fårö / Sweden, 10.-12. August 2004, pp. 6.3.16.3.11.

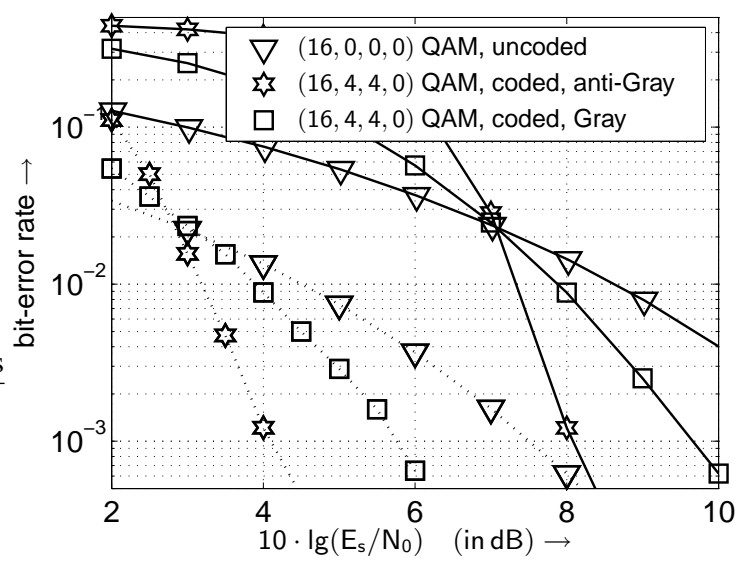

Fig. 11. BER with perfect CSI (dotted line) and imperfect CSI (solid line) assuming different mapping schemes, $\nu=3$ iterations and an effective user throughput of $4 \mathrm{bit} / \mathrm{s} / \mathrm{Hz}$ at a normalized Doppler frequency of $f_{\mathrm{d}} T_{\mathrm{s}}=10^{-3}$

[9] I. Kalet, "Optimization of Linearly Equalized QAM," IEEE Transactions on Communications, vol. 35, no. 11, pp. 1234 1236, November 1987.

[10] J. G. Proakis, Digital Communications., McGraw-Hill, Boston, 2000.

[11] G. D. Forney, R. G. Gallager, G. R. Lang, F. M. Longstaff, and S. U. Qureshi, "Efficient Modulation for Band-Limited Channels," IEEE Journal on Selected Areas in Communications, vol. 2, no. 5, pp. 632-647, 1984.

[12] I. Kalet, "The Multitone Channel.," IEEE Transactions on Communications, vol. 37, no. 2, pp. 119-124, Februar 1989.

[13] L. Hanzo and T. Keller, OFDM and MC-CDMA, Wiley, New York, 2006

[14] Z. Luo, H. Gao, Y. Liu, and J. Gao, "Robust pilot-symbolaided MIMO channel estimation and prediction.," in IEEE Global Telecommunications Conference, Dallas, Texas, USA, 29. November - 3. December 2003, pp. 3646 - 3650.

[15] A. Duel-Hallen, S. Hu, and H. Hallen, "Long Range Prediction of Fading Signals: Enabling Adaptive Transmission for Mobile Radio Channels," IEEE Signal Processing Magzine, vol. 17, no. 3, pp. 62-75, May 2000.

[16] S. Coleri, M. Ergen, A. Puri, and A. Bahai, "Channel Estimation Techniques based on Pilot Arrangement in OFDM Systems," IEEE Transactions on Broadcasting, vol. 48, no. 3, pp. 223-229, May 2002, September.

[17] L. Hanzo, S. X. Ng, W. Webb, and T. Keller, Quadrature Amplitude Modulation: From Basics to Adaptive Trellis-Coded, Turbo-Equalised and Space-Time Coded OFDM, CDMA and MC-CDMA Systems, Wiley, New York, 2004.

[18] J. A. Chindapol, A. Ritcey, "Design, Analysis, and Performance Evaluation for BICM-ID withsquare QAM Constellations in Rayleigh Fading Channels," IEEE Journal on Selected Areas in Communications, vol. 19, no. 5, pp. 944-957, May 2001. 